\title{
High-efficiency, long-pulse operation of MW-level dual-frequency gyrotron, 84/126GHz, for the TCV Tokamak
}

\author{
S. Alberti ${ }^{1}$, K. A. Avramidis ${ }^{2}$, W. Bin ${ }^{3}$, A. Bertinetti ${ }^{4}$, J. Dubray ${ }^{1}$, D. Fasel ${ }^{1}$, S. Garavaglia ${ }^{3}$, J. \\ Genoud $^{1}$, T. Goodman ${ }^{1}$, J. P. Hogge ${ }^{1}$, P.-F. Isoz ${ }^{1}$, P. Lavanchy ${ }^{1}$, F. Legrand ${ }^{5}$, B. Marlétaz ${ }^{1}$, J. Masur ${ }^{1}$, \\ A. Moro ${ }^{3}$, I. Gr. Pagonakis ${ }^{2}$, M. Silva ${ }^{1}$, U. Siravo ${ }^{1}$, M. Toussaint ${ }^{1}$ \\ ${ }^{1}$ Swiss Plasma Center, EPFL Lausanne, CH-1015, Switzerland. \\ ${ }^{2}$ IHM, Karlsruhe Institute of Technology, 76131 Karlsruhe, Germany. \\ ${ }^{3}$ Institute of Plasma Physics "P. Caldirola”, National Research Council of Italy, Milano, Italy \\ ${ }^{4}$ Politecnico Di Torino (POLITO), Corso Duca degli Abruzzi, 24 10129, Torino, Italy. \\ ${ }^{5}$ Microwave Imaging Solution, THALES Vélizy-Villacoublay, F-78141, France.
}

\begin{abstract}
The first unit of the dual-frequency gyrotron, 84$126 \mathrm{GHz} / 1 \mathrm{MW} / 2 \mathrm{~s}$, for the upgrade of the TCV ECH system has been delivered and is presently being commissioned. During a first phase, long-pulse operation $\left(\mathrm{T}_{\mathrm{RF}}>0.5 \mathrm{~s}\right)$ has been achieved and powers in excess of $0.93 \mathrm{MW} / 1.1 \mathrm{~s}$ and $1 \mathrm{MW} / 1.2 \mathrm{~s}$ have been measured in the evacuated RF-load at the two frequencies, $84 \mathrm{GHz}$ ( $T E_{17,5}$ mode) and $126 \mathrm{GHz}$ ( $\mathrm{TE}_{26,7}$ mode), respectively. Considering the different $\mathbf{r f}$ losses in the experimental setup, the power level generated in the gyrotron cavity is in excess of $1.1 \mathrm{MW}$ and 1.2MW, with a corresponding electronic efficiency of $35 \%$ and $36 \%$. These values are in excellent agreement with the design parameters and would likely lead to a gyrotron total efficiency higher than $50 \%$ in case of implementation of a depressed collector. The gyrotron behavior is remarkably reliable and robust with the pulse length extension to $2 \mathrm{~s}$ presently only limited by external auxiliary systems.
\end{abstract}

\section{INTRODUCTION}

$\mathrm{T}$ HE X3 upgrade project [1] of the Swiss Plasma Center consists of adding two dual-frequency gyrotrons (126 or $84 \mathrm{GHz} / 1 \mathrm{MW} / 2 \mathrm{~s}$ ) with a total power of $2 \mathrm{MW}$ at $126 \mathrm{GHz}$ (for top-launch (TL) X3-mode) or at $84 \mathrm{GHz}$ (for low field side (LFS) injection X2-mode). The gyrotron design is the result of a collaborative effort between SPC and KIT and its manufacturing is made by theThales.

The tube design is largely based on the existing Thales TH1507 $140 \mathrm{GHz} / 1$ MW gyrotron developed by KIT and SPC for W7-X [2,3], for which some components have been modified either to include state of the art design criteria or to optimize the operation at the two different frequencies. This encompasses the use of a triode gun instead of a diode gun to keep control of the electron beam parameters at both frequencies, an increase of the cavity length to ensure an optimized interaction length at the two frequencies, a hybridtype launcher designed to provide a high output beam gaussian content for the two modes of operation, an adjustable last mirror to center the output beam at the window in case of necessity, an optimized collector cooling geometry and a diamond window thickness adapted at 84 and $126 \mathrm{GHz}$.

The first unit has been manufactured by Thales and delivered in the third quarter 2018 [4]. The pulse length extension to $2 \mathrm{~s}$ is ongoing and presently limited by external auxiliaries.

\section{EXPERIMENTAL RESULTS}

Short-pulse ( $\leq 20 \mathrm{~ms})$ and long-pulse operation $(\geq 500 \mathrm{~ms})$ has been successfully achieved during the commissioning phase without facing any issues. For long-pulse operation, a novel power deposition scheme on the gyrotron collector, based on an AM modulation of the collector sweeping coil, has been fully validated and ensures an homogenous power distribution along the collector walls [5]. The experimental set-up during commissioning is shown in Fig.1.

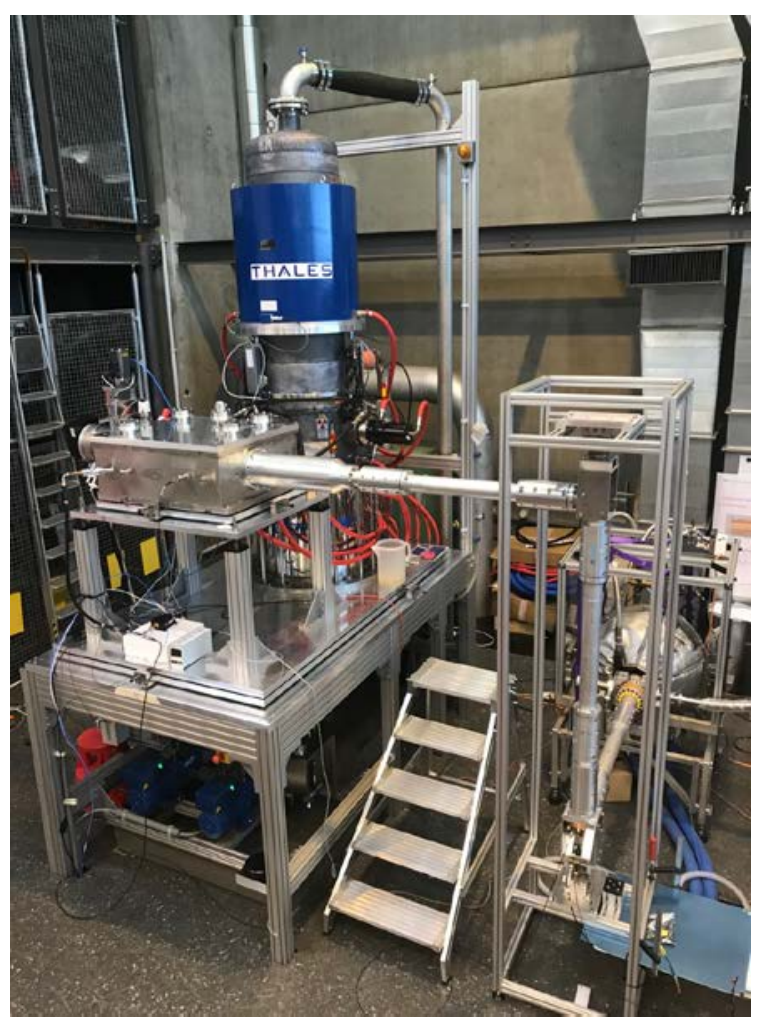

Fig.1. Layout of the experimental setup used for long-pulse characterization of the dual-frequency gyrotron. Via an evacuated MOU (rectangular metallic box), the rf-power at the two frequencies is transmitted to the evacuated high-power load [6] using a $\mathrm{HE}_{11}(63.5 \mathrm{~mm}$ diameter) evacuated waveguide. 
During the commissioning, the gyrotron optimization was guided by extensively using the modeling tools available at SPC $[7,8]$. In long-pulse operation $\left(\mathrm{T}_{\mathrm{RF}}>0.5 \mathrm{~s}\right)$ stable monomode operation was reached and the cavity generated rf-power is well in excess of $1 \mathrm{MW}$ at the two frequencies with corresponding electronic efficiencies higher than $35 \%$. A summary of the achieved gyrotron performances is given in Table 1 .

TABLE 1. Achieved long pulse ( $\geq 500 \mathrm{~ms}$ ) performance of the gyrotron.

\begin{tabular}{lll}
\hline Parameter & X2 & X3 \\
\hline Frequency [GHz] & 84 & 126 \\
Mode $\mathrm{TE}_{\mathrm{m}, \mathrm{p}}$ & $\mathrm{TE}_{+17,6}$ & $\mathrm{TE}_{+26,7}$ \\
Beam current $\mathrm{I}_{\mathrm{b}}[\mathrm{A}]$ & 40 & 42 \\
Cathode voltage $\mathrm{V}_{\mathrm{k}}[\mathrm{kV}]$ & 80 & 81.5 \\
Beam energy $\mathrm{V}_{\mathrm{b}}[\mathrm{kV}]$ & 78 & 79.5 \\
Anode voltage $\mathrm{V}_{\mathrm{a}}[\mathrm{kV}]$ & 44 & 55.5 \\
$\mathrm{P}_{\mathrm{RF}}$ load [MW] & 0.93 & 1.04 \\
Load reflection [\%] (est.) & 4 & 4 \\
TL losses (5 mirrors) [\%] (est.) & 0.8 & 0.8 \\
Internal mirr. losses [\%] (est.) & 0.3 & 0.3 \\
Launcher losses [kW] (meas.) & 16 & 20 \\
Cavity losses [kW] (meas.) & 32 & 42 \\
Stray radiation [kW] (calc.) & 60 & 40 \\
Cavity RF-power [MW] & $1.09 \pm 5 \%$ & $1.2 \pm 5 \%$ \\
Electronic efficiency [\%] & $35 \pm 1.5$ & $36 \pm 1.5$ \\
Frequency shift [MHz] & 180 & 350 \\
\hline
\end{tabular}

Time traces of the relevant quantities for the two frequencies are shown in Fig.2\&3.
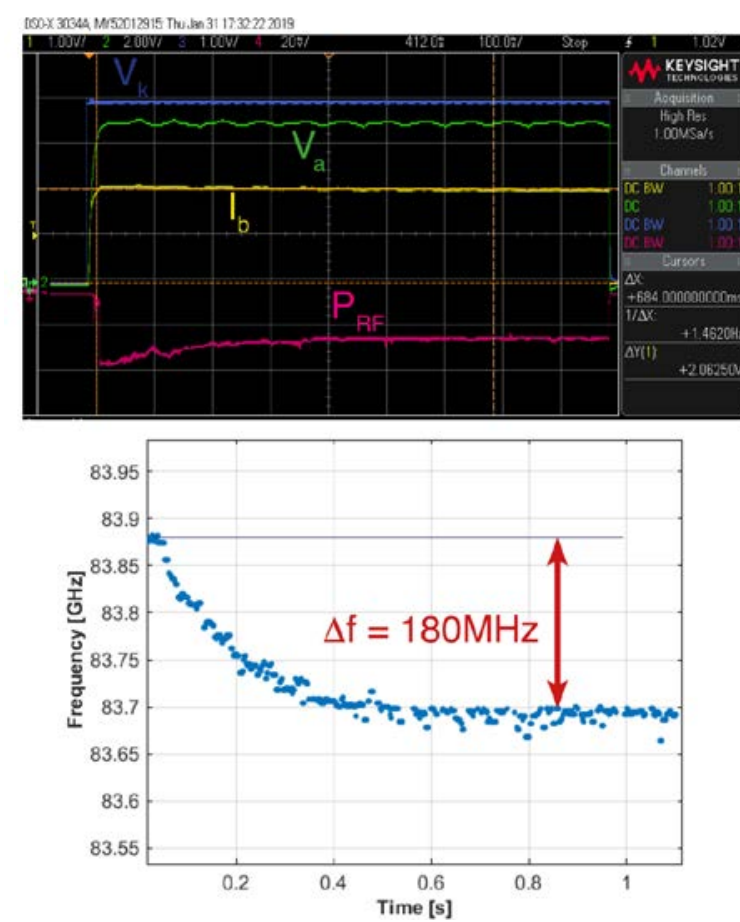

Fig.2. For the operational point @84GHz shown in Table 1, on top, time traces of the electron beam-parameters and RF-power (Schottky diode). Bottom, frequency time evolution.
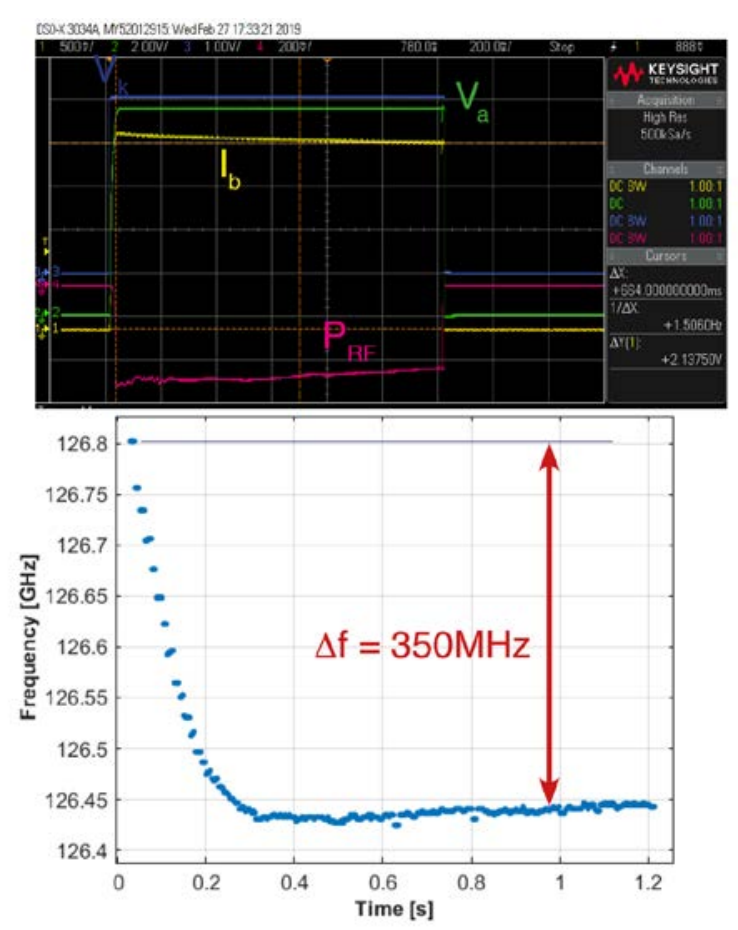

Fig.2. For the operational point @126GHz shown in Table 1, on top, time traces of the electron beam-parameters and RFpower (Schottky diode). Bottom, frequency time evolution.

\section{CONCLUSIONS}

The first dual-frequency gyrotron at $84 \mathrm{GHz}$ and $126 \mathrm{GHz}$ for TCV has been delivered by Thales and commissioned at SPC. The nominal power of $1 \mathrm{MW}$ has been reached at both frequencies, with electronic efficiencies of the order of $35 \%$, suggesting that an efficiency of $50 \%$ could be reached if a depressed collector was used. The specific design options increased cavity length, triode gun, hybrid-type launcher, improved collector cooling and sweeping scheme - have been validated. An excellent agreement was found between the experimental observations and numerical simulations performed with the monomode code TWANG. In addition, no parasitic oscillation has been detected. The extension to the nominal pulse length $(2 \mathrm{~s})$ will be carried out once present limitations have been overcome. The second gyrotron will be delivered in July 2019 and the first operation on TCV is foreseen during the $2^{\text {nd }}$ half of 2019.

\section{ACKNOWLEDGMENTS}

This work is partially supported by the Swiss National Science Foundation.

\section{REFERENCES}

[1] Fasoli A. for the TCV team, Nucl. Fusion, 55 (2015) 043006.

[2] S. Alberti et. al., Fusion Engineering and Design 53, 387 (2001).

[3] J. Jelonnek et. al.,IEEE Trans. on Plasma Science 42(5), 1135 (2014).

[4] R. Marchesin et. al., Proc. of IEEE IVEC 2019, Busan, South Korea.

[5] J.-Ph. Hogge et al., Proc. of RFPPC 2019, Hefei, China.

[6] W. Bin et al., Fusion Eng. Des., doi.org/10.1016/j.fusengdes.2018.11.019

[7] F. Braunmueller et al., Physics of Plasmas, 22(6), 063115 (2015).

[8] J. Genoud et al., Physics of Plasmas, 23(4), 043101 (2016). 